\title{
QCD at high density/temperature
}

\author{
M. Alford ${ }^{\mathrm{a}}$ \\ a Physics and Astronomy Department \\ Glasgow University \\ Glasgow G12 8QQ \\ U.K. \\ I summarize recent work on QCD at high temperature and density.
}

\section{INTRODUCTION}

QCD now occupies an established position as the well-attested theory of strong-interaction physics. Other contributions to these proceedings (those of S. Frixione, L. Lellouch, and K. Long) survey the agreement between evidence from particle collider experiments and perturbative and lattice QCD calculations.

However, there is more to QCD than collisions between pairs of particles. QCD should also be able to tell us about the thermodynamics of matter in the realm of extraordinarily high temperatures $(\gtrsim 100 \mathrm{MeV})$ and densities at which it comes to dominate the behavior. This regime has real physical importance: the whole universe was hotter than $100 \mathrm{MeV}$ for the first crucial microseconds of its history, and in the current epoch our galaxy has a large population of neutron stars consisting of matter squeezed beyond nuclear density by gravitational forces. However, only in the last few years have these regimes begun to be probed experimentally in heavy ion collisions and astrophysical observations of neutron stars, and we are still working towards achieving a proper theoretical understanding of them.

In this paper I will survey the progress that has been made in the last few years in qualitative predictions and quantitative calculations of the properties of QCD at very high temperature and net baryon density. I will not discuss theory that is specifically tailored to heavy ion collisions, but refer the reader to existing review articles [1]. Other more tightly focused reviews of QCD at

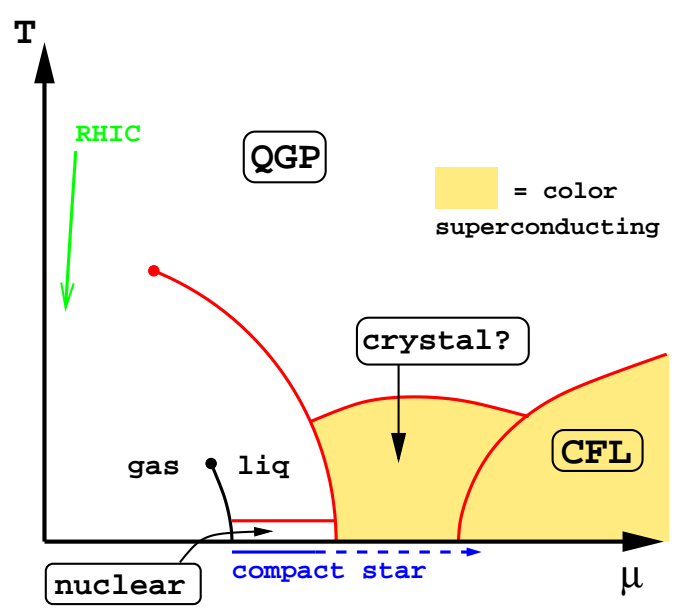

Figure 1. A conjectured phase diagram for QCD in the real world. The CFL region may alternatively extend down to the nuclear phase, with no intermediate phase. Depending on the strength of instanton interactions, the CFL phase may include $K^{0}$ condensation.

high density [2] or high temperature [3] may also prove useful for details that go beyond the scope of this summary.

\section{Phases of QCD}

Fig. 1 shows a conjectured phase diagram for QCD. Along the horizontal axis the temperature is zero, and the density rises from the onset of 
nuclear matter through the transition to quark matter. Compact stars are in this region of the phase diagram, although it is not known whether their cores are dense enough to reach the quark matter phase. Along the vertical axis the temperature rises, taking us through the crossover from a hadronic gas to the quark gluon plasma. This is the regime explored by high-energy heavy-ion colliders such as RHIC (see the contribution by J. Harris to these proceedings). At high temperature and moderate density we find the first-order chiral phase transition ending at the chiral critical point [4], which could perhaps be detected [5] in medium-energy heavy ion colliders (see Sect. 5).

For some parts of the phase diagram reliable calculations can be performed. Standard perturbative methods can be used in the outer regions of high density $\left(\mu \gtrsim 10^{8} \mathrm{MeV}[6]\right)$ and temperature $\left(T \gtrsim 10^{387} \mathrm{GeV}[\overline{7}]\right)$, and resummation methods can extend this down to $T \gtrsim 3 T_{c}$ (Section 1.2). At zero density and $T \lesssim 4 T_{c}$, lattice methods give good results, and ways have recently been found to extend lattice methods to $\mu \lesssim T$. However, this leaves much of the most interesting phase structure in regions where controlled calculations are not yet possible. In the following sections, I will describe the progress that has been made in understanding QCD at high density, in achieving first-principles calculations at high temperature, in lattice calculations at high temperature and low density, and in gaining control of field theories out of thermal equilibrium.

\section{High density QCD}

It is difficult to make accurate predictions for high density QCD because lattice gauge theory is stymied by the complexity of the fermion determinant (see Sect. 5). However, we can borrow some intuition from condensed matter physics, which tells us to expect the very interesting phenomenon of color superconductivity [8,9] QCD is asymptotically free - the interaction becomes weaker as the momentum grows - so at sufficiently high density and low temperature, there is a Fermi surface of almost free quarks. The interactions between quarks near the Fermi surface are certainly attractive in some channels (after all, QCD binds quarks together to form baryons) and it was shown by Bardeen, Cooper, and Schrieffer (BCS) 10] that if there is any channel in which the interaction is attractive, then there is a state of lower free energy than a simple Fermi surface. That state arises from a complicated coherent superposition of pairs of particles (and holes) - "Cooper pairs".

Heuristically, the BCS argument is this. The Helmholtz free energy is $F=E-\mu N$, where $E$ is the total energy of the system, $\mu$ is the chemical potential, and $N$ is the number of fermions. The Fermi surface is defined by a Fermi energy $E_{F}=\mu$, at which the free energy is minimized, so adding or subtracting a single particle costs zero free energy. Now switch on a weak attractive interaction. It costs no free energy to add a pair of particles (or holes), and the attractive interaction between them then lowers the free energy of the system. Many such pairs will therefore be created in the modes near the Fermi surface, and these pairs, being bosonic, will form a condensate. The ground state will be a superposition of states with all numbers of pairs, breaking the fermion number symmetry.

Since pairs of quarks cannot be color singlets, the resulting condensate will break the local color symmetry $S U(3)_{\text {color }}$. We call this "color superconductivity". Note that the quark pairs play the same role here as the Higgs particle does in the standard model: the color-superconducting phase can be thought of as the Higgs phase of QCD.

\subsection{Calculation methods}

To decide whether or not fermions condense in the ground state, one can explicitly construct a wavefunctional with the appropriate kind of pairing, and use a many-body variational approach to find the pairing strength by minimizing the free energy. Alternatively but equivalently, one can formulate the problem field-theoretically, in terms of mean-field Schwinger-Dyson equations for a quark self energy that includes "anomalous" $\langle q q\rangle$ terms as well as the usual $\langle\bar{q} q\rangle$ terms. In either case the problem reduces to solving a selfconsistency equation, the gap equation, to find the self energy. If the only solution is zero, there is no condensation in that channel. If not, there 
can be condensation, but it may just be a local minimum of the free energy. There may be other solutions to the gap equation, and the one with the lowest free energy is the true ground state.

There are several possible choices for the interaction to be used in the Lagrangian from which we derive the gap equation. At asymptotically high densities $\left(\mu \gtrsim 10^{8} \mathrm{MeV}\right.$ [6]) QCD is weakly coupled, so one gluon exchange is appropriate. Such calculations 11, 12] demonstrate from first principles that color superconductivity occurs in QCD. However, the density regime of physical interest for neutron stars or heavy ion collisions is up to a few times nuclear density $(\mu \lesssim 500 \mathrm{MeV})$, so one must use a phenomenological interaction that can be argued to capture the essential physics of QCD. The usual choice is an NJL model, with a four-fermion interaction based on known QCD interactions (gluon exchange, or instantons) whose strength can be normalized to reproduce known low-density physics such as the chiral condensate, and then extrapolated to the desired chemical potential. Such calculations 8.9, 13, 14 find that the color superconducting gap is insensitive to the form of the interaction, and rises to values around $100 \mathrm{MeV}$. Various other calculations that include competition between chiral symmetry breaking and quark pairing confirm this [4, 15]. Very recently, the first lattice calculation of a $3+1$ dimensional NJL model with chemical potential has been performed, and there are indications of diquark condensation [16].

The favored pairing pattern at high densities, where many strange quarks are present, is "colorflavor locking" (CFL), which will be described below. At lower densities there may be a direct transition from nuclear matter to CFL quark matter (see Section 3.6). Alternatively, at intermediate densities there may be another phase. One possibility is the "2SC" phase which has $u$ - $d$ pairing only, but this now seems unlikely to occur in equilibrated quark matter (Section 3.3). Other possibilities include crystalline pairing (see Section 3.7), or some sort of single-flavor pairing [17].

\subsection{Three flavors: Color-flavor locking (CFL)}

In $\mathrm{QCD}$ with three flavors of massless quarks the Cooper pairs cannot be flavor singlets, and both color and flavor symmetries are necessarily broken [14]. Both NJL 14, 18,19 and gluonmediated interaction calculations [12] agree that the attractive channel exhibits a pattern called color-flavor locking (CFL) 1,

$$
\begin{aligned}
\left\langle q_{i}^{\alpha} q_{j}^{\beta}\right\rangle & \sim \delta_{i}^{\alpha} \delta_{j}^{\beta}+\kappa \delta_{j}^{\alpha} \delta_{i}^{\beta} \\
{\left[S U(3)_{\text {color }}\right] } & \times \underbrace{S U(3)_{L} \times S U(3)_{R}}_{\supset\left[U(1)_{Q}\right]} \times U(1)_{B} \\
& \rightarrow \underbrace{S U(3)_{C+L+R}}_{\supset\left[U(1)_{\tilde{Q}}\right]} \times \mathbb{Z}_{2}
\end{aligned}
$$

(color indices $\alpha, \beta$ and flavor indices $i, j$ run from 1 to 3$)$. The Kronecker deltas connect color indices with flavor indices, so that the condensate is not invariant under color rotations, nor under flavor rotations, but only under simultaneous, equal and opposite, color and flavor rotations. Since color is only a vector symmetry, this condensate is only invariant under vector flavor+color rotations, and breaks chiral symmetry. The effect of including a sufficiently large strange quark mass is to unpair the strange quark 21, 18,22, and at lower values it may induce a flavor rotation of the condensate known as "kaon condensation" (Ref. 23] and Section 3.11).

The features of this pattern of condensation are

- The color gauge group is completely broken. All eight gluons become massive. This ensures that there are no infrared divergences associated with gluon propagators.

- All the quark modes are gapped. The nine quasiquarks (three colors times three flavors) fall into an $\mathbf{8} \oplus \mathbf{1}$ of the unbroken global $S U(3)$, so there are two gap parameters. The singlet has a larger gap than the octet.

- A rotated electromagnetism (" $\tilde{Q}$ ") survives unbroken. It is a combination of the original photon and one of the gluons.

${ }^{1}$ Interestingly, such a pattern was at one time considered 
- Two global symmetries are broken, the chiral symmetry and baryon number, so there are two gauge-invariant order parameters that distinguish the CFL phase from the QGP, and corresponding Goldstone bosons which are long-wavelength disturbances of the order parameter. If a quark mass were introduced then it would explicitly break the chiral symmetry and give a mass to the chiral Goldstone octet, but the CFL phase would still be a superfluid, distinguished by its baryon number breaking.

- The symmetries of the 3-flavor CFL phase are the same as those one might expect for 3 -flavor hypernuclear matter [18], so it is possible that there is no phase transition between them.

\subsection{Absence of 2SC}

Until recently it was thought that if there were not enough strange quarks present in quark matter, as might happen at intermediate density, the favored pairing pattern would be $2 \mathrm{SC}$, in which the $u$ and $d$ quarks of two colors pair. leaving the $s$ quarks and third color quarks unpaired. But in a real neutron star we must require electromagnetic and color neutrality [24.22] (ignoring charge-separated phases, see Section 3.6). It turns out that this penalizes the $2 \mathrm{SC}$ phase relative to the CFL phase [22]. The reason is that the CFL phase has already paid most of the cost of neutrality, since it brings the $u, d$, and $s$ Fermi surfaces close together [22, 25, 26, and the 2SC phase only pairs 4 of the 9 quark colors and flavors, so it has much less pair binding energy than the CFL phase.

The arguments made in Ref. 22 are modelindependent, based on simplified assumptions about the dependence of the constituent strange quark mass $M_{s}$ on $\mu$ and expanding the free energy in powers of $M_{s} / \mu$. The NJL calculation of Ref. [26] handles $M_{s} \sim \mu$ and includes the coupling between the chiral condensate and quark condensate gap equations. The net result is that once neutrality is imposed, there is no (or very little [26]) density range in which $2 \mathrm{SC}$ is the phase with the lowest free energy.

\subsection{Signatures in compact stars}

The only place in the universe where we expect sufficiently high densities and low temperatures is compact stars, also known as "neutron stars", since it is often assumed that they are made primarily of neutrons. A compact star is produced in a supernova. Typical compact stars have masses close to $1.4 M_{\odot}$, and are believed to have radii of order $10 \mathrm{~km}$. although uncertainty about the equation of state leaves us unsure of the radius and the maximum density attained in the core. During the supernova, the core collapses, and its gravitational energy heats it to temperatures of order $10^{11} \mathrm{~K}$ (tens of $\mathrm{MeV}$ ), but it cools rapidly by neutrino emission. Within a few minutes its internal temperature $T$ drops to $10^{9} \mathrm{~K}(100 \mathrm{keV})$. Neutrino cooling continues to dominate for the first million years of the life of the star. (For detailed treatments and and reviews see Ref. [27]).

Color superconductivity affects the equation of state at order $(\Delta / \mu)^{2}$. It also gives mass to excitations around the ground state: it opens up a gap at the quark Fermi surface, and makes the gluons massive. One would therefore expect its main physical manifestations to relate to transport properties, such as mean free paths, conductivities and viscosities. In the following sections we will survey some of these manifestations.

\subsection{Mass-radius relationship}

Although the effects of color superconductivity on the quark matter equation of state are subdominant, they may have a large effect on the mass-radius relationship. The reason for this is that the pressure of quark matter relative to the hadronic vacuum contains a constant (the "bag constant" $B$ ) that represents the cost of dismantling the chirally broken and confining hadronic vacuum,

$p \sim \mu^{4}+\Delta^{2} \mu^{2}-B$.

If the bag constant is large enough so that nuclear matter is favored (or almost favored) over quark matter at $\mu \sim 320 \mathrm{MeV}$, then the bag constant and $\mu^{4}$ terms almost cancel, and the superconducting gap $\Delta$ may have a large effect on the equation of state and hence on the mass-radius relationship of a compact star [28]. 


\subsection{Interfaces and mixed phases}

If color superconducting quark matter exists in compact stars, we expect it to occupy a core region, surrounded by a mantle of nuclear matter. There may be a sharp interface between the two, but it is well known that a mixed phase region is also possible [29], given that there are multiple chemical potentials in nuclear matter: for baryon number, electric charge, and also color. A mixed phase region would have distinctive characteristics. Its transport properties are very different from those of the uniform CFL state: neutrino mean free paths, which are hundreds of meters in the CFL phase at temperatures of a few $\mathrm{MeV}$ [30], are short in the mixed phase due to coherent scattering [31].

The nature of the boundary between nuclear and CFL quark matter has been studied [32], and it was found that a mixed phase only occurs if the surface tension of the interface is less than about $40 \mathrm{MeV} / \mathrm{fm}^{2}=0.2 \times(200 \mathrm{MeV})^{3}$, a fairly small value compared to the relevant scales $\Lambda_{\mathrm{QCD}} \approx 200 \mathrm{MeV}, \mu \sim 400 \mathrm{MeV}$. This means that there may well be a sharp interface rather than a mixed phase, with a baryon and energy density discontinuity of about a factor of two across it. Such an interface may modify the mass vs. radius relationship for compact stars with quark matter cores. It may also have qualitative effects on the gravitational wave profile emitted during the in-spiral and merger of two compact stars of this type. Finally, it will affect the $r$-mode spectrum (see Section 3.10), and the damping forces to which $r$-modes are subject.

\subsection{Glitches and the crystalline color su- perconductor}

There is a crystalline form of quark pairing (the "LOFF" 33 phase) that occurs when two different types of quark have sufficiently different Fermi momenta (because their masses or chemical potentials are different) that BCS pairing cannot occur 34$]^{2}$.

Such situations are likely to be generic in nature, where, because of the strange quark mass,

\footnotetext{
2 At large number of colors there is the possibility of a separate "chiral crystal" phase [35], which is not a superfluid, so it will not lead to glitches.
}

combined with requirements of weak equilibrium and charge neutrality, all three flavors of quark in general have different chemical potentials.

The phenomenology of the crystalline phase has not yet been worked out, but recent calculations using Landau-Ginzburg effective theory indicate that the favored phase may be a facecentered cubic crystal [36], with a reasonably large binding energy. This raises the interesting possibility of glitches in quark matter stars.

Glitches are sudden jumps in rotation frequency $\Omega$ of a pulsar. They are thought to arise from pinning of superfluid vortices in the nuclear matter core to the lattice of nuclei that forms the crust [37. As the pulsar spins down, the vortices move outwards, and eventually overcome the pinning forces, and rearrange themselves. Until recently it was thought that quark matter stars would not glitch, because quark matter was thought to have no crust or other rigid structure that could pin the vortices. Now, however, we can envisage an alternative scenario for glitches (supported only be dimensional estimates at this point) where a superfluid CFL core is surrounded by a crystalline layer of quark matter in a LOFF phase.

\subsection{Cooling by neutrino emission}

For its first million or so years, a compact star cools by neutrino emission. There are many uncertainties in determining the temperature of a compact star (from its X-ray spectrum) and its age (inferred from the spindown rate) but observers are now able to map out cooling curves [38, 39, 40]. The cooling rate is determined by the heat capacity and emissivity, both of which are sensitive to the spectrum of low-energy excitations, and hence to color superconductivity $38,41,42,30$.

In the CFL phase, all quarks and gluons have gaps $\Delta \gg T$, electrons are absent [25], and the pseudo-Goldstone bosons have masses of order tens of $\mathrm{MeV}$ [43], so the transport properties are dominated by the only true Goldstone excitation, the superfluid mode arising from the breaking of the exact baryon number symmetry. This means that CFL quark matter has a much smaller neutrino emissivity and heat capacity than nuclear 
matter, and hence the cooling of a compact star is likely to be dominated by the nuclear mantle rather than the CFL core 38,42,44. A CFL core is therefore not expected to be detectable by cooling measurements, although it has been suggested that it might slow the first $\approx 100$ years of cooling 41.

Other phases such as 2SC or LOFF give large gaps to only some of the quarks, leaving the others with no gaps (or very small gaps in weakly attractive channels) so they may allow fast cooling, which is severely constrained by the latest space-based X-ray observations [40]. The cooling would proceed quickly, then slow down suddenly when the temperature fell below the smallest of the small weak-channel gaps. If no sign of such behavior is seen as our observations of compact star temperatures improve then we will have to conclude that either these phases do not occur, or their gaps are much larger than we expected.

\subsection{The neutrino pulse at birth}

In the first minute of a supernova, the inner regions cool rapidly, radiating off much of their energy as neutrinos, whose detailed spectrum as a function of time is determined by the neutrino diffusion properties of the protoneutron star, and can be detected in terrestrial experiments such as SuperKamiokande.

Initial calculations by Carter and Reddy 45 found a striking signature: the core released few neutrinos when it was close to the critical temperature for color superconductivity $T \approx T_{c}$, then a late blast of neutrinos as their mean free path grew larger than the size of the star at $T \ll T_{c}$. This picture is complicated by scattering of neutrinos by Goldstone bosons [30]. Also, the temporal variation of neutrino emission from the core over the first few seconds is liable to be blurred by interactions with the surrounding material. Detailed numerical simulations of core collapse, including the effects of quark pairing, are now needed.

\subsection{0. r-mode instability}

An " $r$-mode" (short for "rotational mode") [46] is a bulk flow in a rotating star that radiates away energy and angular momentum in the form of gravitational waves (for a review, see Ref. [47]). If the rotation frequency of the star is above a critical value, the system becomes unstable to $r$-modes and quickly spins down to the critical value, at which point the $r$-modes are damped out. The critical frequency depends on the sources of damping, such as shear and bulk viscosities, and also friction at the interface between the $r$-mode region and adjacent region. One can therefore rule out certain models for compact stars on the grounds that they have such low damping that they could not support the high rotation rates observed in pulsars: $r$-mode spindown would have slowed them down.

It is generally believed that ordinary nuclear matter is not ruled out in this way [48], but Madsen 449 has shown that color superconductivity, by creating gaps in the quark excitation spectrum, suppresses the viscosities by factors of order $\exp (-\Delta / T)$, encouraging $r$-mode spindown. Furthermore, since CFL matter contains no electrons [25], there is no electrostatic cushion to support a crust of normal matter, which would otherwise have been an additional source of friction. Madsen found that for a compact star made entirely of quark matter in the CFL phase, even a gap as small as $\Delta=1 \mathrm{MeV}$ is ruled out by observations of millisecond pulsars.

It remains to extend this calculation to the more generic picture of a quark matter core surrounded by a nuclear mantle. The friction at the core-mantle interface may be enough 448,49 to quash the $r$-modes. Finally, quark matter may contain a region of LOFF crystal, and the $r$ modes could be damped at the edges of that region rather than at the crust.

\subsection{Other phenomenology}

Many other physical phenomena associated with color superconductivity in quark matter have been investigated. Small chunks of quark matter ("strangelets") may exist, and standard calculations must be modified in that context to account properly for finite volume effects [50]. Strangelets in the CFL phase will have a different mass-charge relationship from those made of unpaired quark matter [51]. At high enough densities $U(1)_{A}$ breaking is suppressed, leading to do- 
main walls bounded by strings [52].

There are many interesting questions about the influence of the strange quark mass on color superconductivity. When $m_{s}=0$ the favored phase is CFL, as discussed in Section 3.2. As the strange quark mass is increased from zero the CFL pairing becomes harder and harder to maintain, and at some point it ceases to be the ground state 21. But before that the system may respond to the finite $m_{s}$ by rotating the quark-quark condensate in flavor space, in a direction that reduces the number of strange quarks. This can be understood as condensation of kaons 23, where by "kaon" we mean the pseudo Goldstone bosons that are associated with the breaking of the chiral flavor symmetry by the CFL condensate. The resultant CFL- $\mathrm{K}^{0}$ phase would break isospin and may also include charged kaons in the first seconds after the supernova, before neutrinos escape the star [53,54].

It is unclear whether kaon condensation will occur in dense quark matter cores of compact stars. Instanton and electromagnetic interactions tend to disfavor CFL- $\mathrm{K}^{0}$ over ordinary $\mathrm{CFL}$, and the magnitude of instanton effects at the densities of interest is very uncertain [55. But CFL- $\mathrm{K}^{0}$ has many interesting features. It supports topological or near-topological solitons: superconducting Kstrings [56] and domain walls [57] whose possible observable manifestations are yet to be investigated.

\section{High temperature QCD}

We now turn from the horizontal axis of the QCD phase diagram (Fig. 11) to the vertical axis, and study QCD at high temperature but low (net) baryon number density. The standard techniques are lattice gauge theory, perturbation theory, and a hybrid of the two that is generally known as "dimensional reduction".

\subsection{Lattice gauge theory}

Lattice gauge theory is a brute-force evaluation of the functional integral over gauge and quark field configurations that is the theoretical definition of QCD. It is therefore a fully nonperturbative first-principles calculation of the predictions of QCD. To be numerically tractable, the integral must be formulated in Euclidean time,

$$
\begin{aligned}
& Z= \int \mathcal{D} A \mathcal{D} \bar{\psi} \mathcal{D} \psi \exp (-\bar{\psi} M[A] \psi) \\
& \exp \left(-S_{\text {gauge }}[A]\right) \\
&=\int \mathcal{D} A \operatorname{det}(M[A]) \exp \left(-S_{\text {gauge }}[A]\right)
\end{aligned}
$$

where the fermionic matrix $M$ is the usual fermion action

$M[A]=\gamma^{\mu} D_{\mu}+m$

In general, lattice gauge calculations require large computational resources. A typical calculation is formulated in a box of size $V \approx(3 \mathrm{fm})^{3}$, with lattice spacing $a \sim 0.1 \mathrm{fm}$, and with up and down quark masses $m_{u, d} \gtrsim m_{s} / 4$. To obtain physically relevant results it is necessary to extrapolate $a \rightarrow 0, V \rightarrow \infty, m_{u, d} \ll m_{s}$. In addition, not all quantities are accessible to lattice calculations: spectral functions and transport coefficients are defined in Minkowski space, and can only be obtained by some sort of analytic continuation from the lattice results [58]. In spite of these challenges, lattice gauge calculations have been very successful in obtaining predictions for the equation of state of QCD at high temperature.

In a lattice calculation, thermal averages are obtained by setting the length of the Euclidean time direction to the inverse temperature. To probe physics near the deconfinement temperature $T_{c} \sim 200 \mathrm{MeV} \sim(1 \mathrm{fm})^{-1}$ therefore requires a lattice spacing $a \lesssim 0.2 \mathrm{fm}$. To probe much higher temperatures would require a correspondingly smaller lattice spacing, increasing the numerical effort. Thus calculations are so far limited to $T \lesssim 4 T_{c}$. (This restriction may be eased in the future by the introduction of anisotropic lattices, which are finer in the $t$-direction.) State-ofthe-art lattice calculations use improved actions, which include extra terms to compensate for the $\varnothing\left(a^{2}\right)$ discretization errors, and yield continuumlike results from relatively coarse $(a \lesssim 0.2 \mathrm{fm})$ lattices. This is crucial because the cost of a lattice calculation rises as a very high power of $1 / a$. Using improved staggered [59 and Wilson 60 actions the equation of state of QCD has been calculated and is shown in Fig. 2 . 


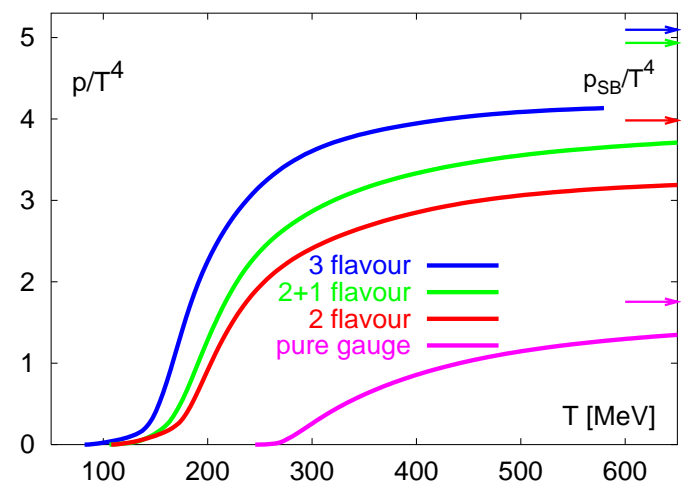

Figure 2. The pressure in QCD with various numbers of dynamical quarks, as a function of temperature. Arrows show the corresponding noninteracting quark+gluon gas pressure $p_{S B}$. For the $(2+1)$-flavor calculation the light quark mass was $m_{u, d} \approx \frac{1}{4} m_{s}$ [59].

The fact that at $T \sim 4 T_{c}$ the pressure is about $15 \%$ less than the pressure $p_{S B}$ of free quarks and gluons shows that there are still important interactions at that temperature. The major challenge for other approaches is to confirm and explain this.

\subsection{Perturbation theory}

Perturbation theory is generally applicable when the coupling is small. One might expect that quarks and gluons at temperature $T$ would be described by QCD with running coupling $g(T)$, which becomes small at high temperature, causing perturbation theory to converge quickly. In fact, this does not in general happen. For example, attempts to calculate the free energy to order $\mathcal{O}\left(g^{5} T^{4}\right)$ [7,61,62] find that there are large corrections with alternating signs, making the naive perturbative series useless for physically interesting temperatures (for illustration, the $g^{3}$ prediction is shown in Fig. 3). As we will see below, there are resummations of the perturbative series that greatly improve its convergence. There are also now results to leading order in $g$ for transport coefficients, whose expansion contains powers of $\log (g)$ and therefore converges even more slowly.

\subsubsection{Equation of state}

There have been various proposals for reorganizing the high-temperature perturbative series for the pressure, in order to improve its convergence. They involve resumming the hard thermal loops (HTL), typically via the CornwallTouboulis-Jackiw two-particle irreducible (2PI) effective action [63], in which the complete propagator is used as an infinite set of variational parameters 664,65. This has been used in approximately self-consistent HTL resummation calculations of the entropy by Blaizot, Iancu and Rebhan (BIR) [66] and of the pressure by Peshier [67]. In a different approach, Andersen, Braaten, Petitgirard, Strickland (ABPS) used the HTL effective action with the Debye screening mass as a variational parameter [68]. The results of such calculations are shown in Fig. 3. The dashed lines show $\varnothing\left(g^{3}\right)$ naive perturbation theory, to illustrate the poor convergence of the perturbative series. (The $\varnothing\left(g^{2}\right)$ and $\varnothing\left(g^{4}\right)$ predictions lie below the free gas result.) The theoretical uncertainty comes from varying the renormalization scale by a factor of 2 around the first Matsubara frequency $2 \pi T$. The grey bands show the results of lattice calculations [69]. Finally, the range of predictions of the BIR and ABPS hard thermal loop methods are shown. The BIR prediction has a bigger theoretical uncertainty because they choose a scale to separate hard and soft contributions, and varying that scale contributes to the error. BIR are currently working to reduce this uncertainty.

We conclude that there are (perhaps somewhat ad hoc) resummations of perturbation theory that indicate that the pressure should slowly approach the free QGP value $P_{S B}$ from below. This is in agreement with, but not yet as accurate as, the lattice calculations. At this point it appears difficult to push these calculations to higher order and thereby judge their validity.

\subsubsection{Transport properties}

The photon emission rate from a quark-gluon plasma has been perturbatively estimated by various groups [71,72,73 to leading order in the 


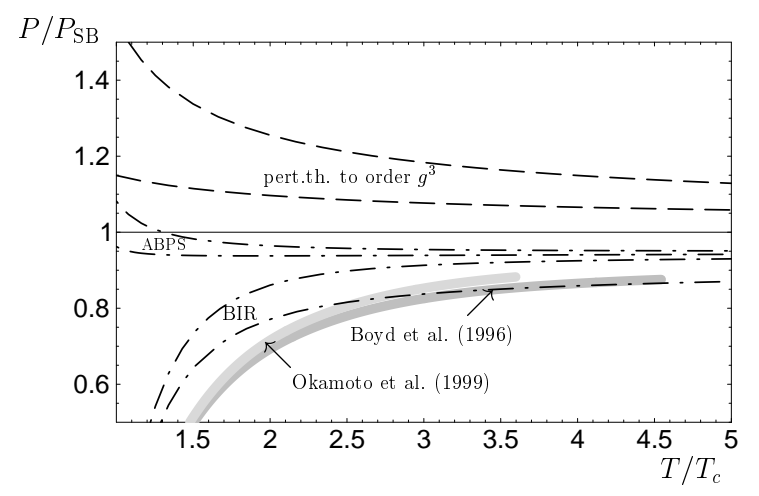

Figure 3. Results 70 of calculations of the pressure of hot gluons, from lattice calculations (Grey bands) and $\varnothing\left(g^{3}\right)$ naive perturbation theory (dashed lines). The dot-dashed lines show the range of predictions from the HTL reorderings of the perturbative series formulated by Blaizot, Iancu, and Rebhan (BIR), and Andersen, Braaten, Petitgirard, and Strickland (ABPS).

electromagnetic and strong coupling constants. However, there are complications due to leading $\operatorname{logs}$ that arise from multiple scattering of co-linear bremsstrahlung (Landau-PomeranchukMigdal (LPM) effect). Only recently have these been fully taken into account by Arnold, Moore, and Yaffe [74. They found that the soft $(k \lesssim 2 T)$ photon emission rate behaves as $d \Gamma / d k \propto \widetilde{k^{-1 / 2}}$, which is less infrared singular than the result $d \Gamma / d k \propto k^{-1}$ obtained in earlier calculations that neglected the LPM effect 73,75. Similar methods are applicable to the dilepton rate, which is an important observable in heavy-ion collisions, and also to transport properties such as the shear viscosity, electrical conductivity, and flavor diffusion constant, which are in principle important input parameters to phenomenological models of the early stages of a heavy-ion collision. We can therefore look forward perturbative calculations of these quantities to leading order in $g$ in the near future.

\subsection{Dimensional reduction}

The intuition behind dimensional reduction is related to the poor behavior of the perturbative expansion in QCD at high temperature, which can be understood by considering the $T \rightarrow \infty$ limit of the pure gauge theory. At non-zero $T$ the Euclidean time size is finite, so the glue fields can be Fourier-analyzed into Matsubara modes, with frequencies $\omega=0,2 \pi T, 4 \pi T$, etc. As $T \rightarrow$ $\infty$, the Euclidean time size goes to zero, and the $\omega=0$ Matsubara "static mode" dominates. The theory reduces to a three-dimensional theory of gluons (the spatial components $A_{i}$ ) and a scalar that transforms as a color octet $\left(A_{0}\right)$ 62, 76,77 . $78,79,80$

$\mathcal{L}=\frac{1}{2} \operatorname{tr} F_{i j}^{2}+\operatorname{tr}\left[D_{i}, A_{0}\right]^{2}+m_{D}^{2} \operatorname{tr} A_{0}^{2}+\lambda_{A}\left(\operatorname{tr} A_{0}^{2}\right)^{2}(5)$

This effective theory is confining, which indicates that the lowest Matsubara mode contains the non-perturbative physics 81,82. It therefore makes sense to perturbatively integrate out the higher Matsubara modes, and obtain a 3D effective action for the static mode. This can then be treated by lattice methods.

The final result for the pressure takes the form of an integral over a coupling constant in the 3D theory, with a parameter $e_{0}$ associated with the constant of integration which can be determined by an arduous 4 -loop calculation in the $4 \mathrm{D}$ theory.

In Fig. 10 we see the range of predictions for the pressure of hot QCD with no quarks for various values of the constant $e_{0}$. The value $e_{0}=10(1)$ agrees best with $4 \mathrm{D}$ lattice calculations. The effort to calculate $e_{0}$ independently is currently underway.

This method is certainly better behaved than $4 \mathrm{D}$ perturbation theory. It may also avoid some of the difficulties that beset $4 \mathrm{D}$ lattice calculations. For example, light quarks only come in through the coefficients in the 3D action, and so do not impose any additional computational burden. Also it is possible to perform calculations at $\mu>0$ [84]. But it also inherits some of their limitations, such as being restricted to thermodynamic observables that can be measured in Euclidean time. 


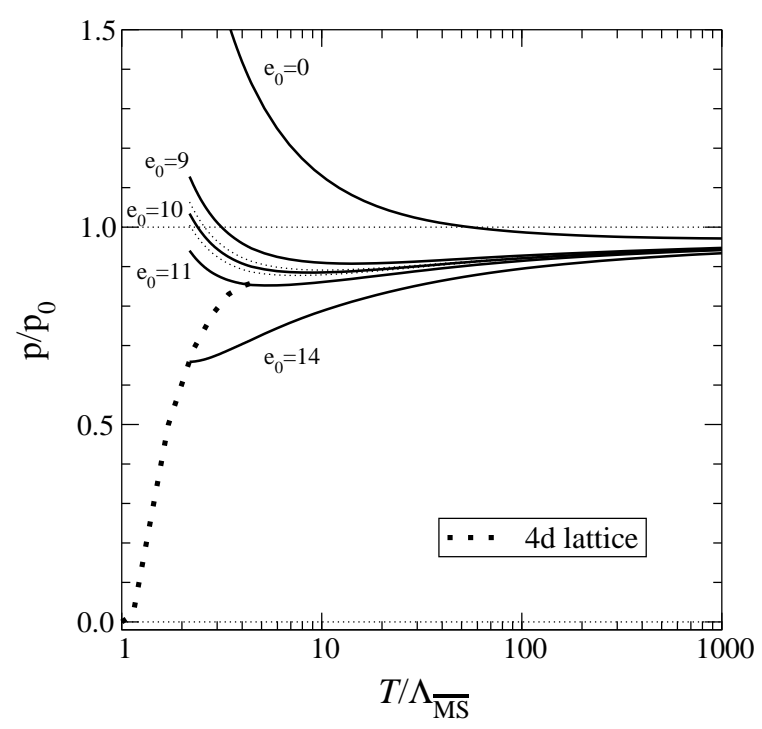

Figure 4. The pressure of hot gluons, calculated by dimensional reduction, for various values of the undetermined constant $e_{0}$ 83. Statistical errors are shown for $e=10$. The dotted line indicates predictions of lattice calculations.

\section{Lattice QCD at high temperature and low density}

Lattice calculations at non-zero density (chemical potential $\mu>0$ ) have to contend with the "sign problem". Until recently this meant that there were no lattice results for realistic ( 3 color, $3+1$ dimensional) QCD away from the $\mu=0$ axis of the phase diagram. There has been a lot of work on more tractable variants such as QCD with two colors 85, QCD at finite isospin density [86], Gross-Neveu models in $2+1$ dimensions 87] and so on. It has also been suggested that at asymptotically high density the sign problem in QCD might become more tractable [88].

In the last few years this situation has changed, with three groups reporting lattice results for the position of the crossover line and even the critical point in the high- $T$ low- $\mu$ region of the QCD phase diagram. Before discussing those results, I will review the nature of the sign problem.
The sign problem is not a deep problem of principle, but it is a very inconvenient feature of the standard formulation of the QCD functional integral. The problem is that when $\mu \neq 0$, the factor of $\operatorname{det}(M[A])$ in the gauge field functional integral (3) is no longer a positive real number. This means that the only practical way of evaluating such a high-dimensional integral, the Monte-Carlo importance sampling method, which interprets the Boltzmann weight $B[A]=$ $\operatorname{det}(M[A]) \exp \left(-S_{\text {gauge }}\right)$ as a probability, cannot be applied.

In principle it might seem that the sign problem could be straightforwardly fixed by "reweighting" 89. One example of this would be to split off the phase of the weight by writing $B[A]=$ $|B[A]| \exp (i \phi[A])$, and use the absolute value of the Boltzmann factor for importance sampling,

$$
\begin{aligned}
Z_{\|} & =\int \mathcal{D} A|B[A]| \\
\langle O\rangle_{\|} & =\left(1 / Z_{\|}\right) \int \mathcal{D} A O[A]|B[A]|
\end{aligned}
$$

The true expectation value of the observable can be obtained by including the complex phase in the observable, taking its expectation value in the $|B|$ ensemble, and dividing by a reweighting factor

$\langle O\rangle=\langle O \exp (i \phi[A])\rangle_{\|} /\left(Z / Z_{\|}\right)$.

But the reweighting factor is the ratio of two partition functions, so it is related to the difference between their free energy densities $f$

$Z / Z_{\|}=\exp \left(-V\left(f-f_{\|}\right)\right)$.

The true partition function $Z$ contains the phase factor $\exp (i \phi[A])$, and is therefore smaller than $Z_{\|}$, so $f>f_{\|}$. Thus the reweighting factor in (7) becomes exponentially small in the thermodynamic limit $V \rightarrow \infty$. The same is true for $\langle O\rangle_{\|}$, because $\langle O\rangle$ itself is not exponentially large in $V$. This means exponentially large statistics are required to obtain any given accuracy in $\langle O\rangle$.

It must be stressed that the sign problem arises from the way we structure our evaluation of the functional integral, integrating out the fermion fields for each fixed gauge field configuration. The physically relevant quantities such as $Z$ and $\langle O\rangle$ are real and positive, and if we could restructure the ensemble of gauge field configurations 
into families within which the weights and observables analytically added up to positive values then there would be no sign problem. This is what cluster algorithms can do, and such a restructuring has been achieved for the $\mathbb{Z}_{3}$ model that approximates QCD at very high quark mass and chemical potential 90]. One strategy for the ultimate solution of the sign problem is to recast the QCD functional integral into a form to which cluster algorithms can be applied 91.

In the meantime, it makes sense to try to perform calculations using the methods available, in regions of the phase diagram where the sign problem is weakest. This is the region of small $\mu$ and high temperature. In the last few years, various groups have have been exploring techniques applicable at $\mu \lesssim T$ : multi-parameter reweighting, derivatives with respect to $\mu$ at $\mu=0$, and imaginary chemical potential.

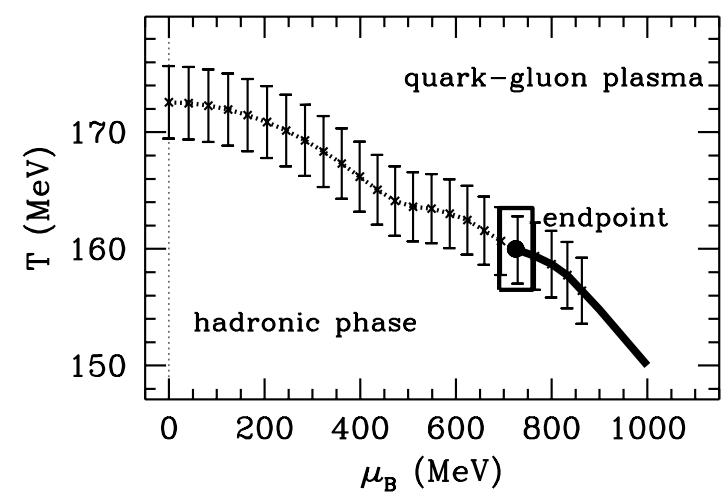

Figure 5. The position in the $\mu-T$ plane of the crossover between the hadronic and QGP phases, including an estimate of the position of the critical point at $\left(\mu^{*}, T^{*}\right)$ and the first-order line, obtained from a lattice calculation with twoparameter reweighting [92]. Note that the horizontal axis is $\mu_{B}=3 \mu$.

\subsection{Multi-parameter reweighting.}

Fodor and Katz 93 suggested a reweighting algorithm like the one described above, but using the $\mu=0$ ensemble to measure observables rather than the $|B|$ ensemble. They exploit the freedom to vary the temperature of the $\mu=0$ ensemble, and thereby improve its overlap with the desired $\mu>0$ ensemble. This only postpones the sign problem to larger volumes, but from their initial results it appears that reasonable results may now be obtained on lattices that are large enough to be physically interesting. Their estimate of the crossover line and chiral critical point is shown in Fig. 5 (for the equation of state see 94 ). The numerical values should be interpreted with caution: the light quark masses are well above their physical values, so the true critical point is presumably at a somewhat lower chemical potential 95. The lattice quark action is unimproved (KogutSusskind) and used at a relatively coarse lattice spacing $a \sim 0.2 \mathrm{fm}$ where it is known to have large discretization errors. Thus there are potentially large systematic errors, and as with all reweighting methods the statistical errors can be misleadingly small. Nonetheless, this is the only method that has obtained the position of the chiral critical point, and it seems there is a real chance that it may be able to do so for physical quark masses. This is a striking improvement over the situation two years ago.

\section{2. $\partial / \partial \mu$ at $\mu=0$.}

A closely related strategy for extending lattice QCD to small chemical potential is to evaluate derivatives of observables with respect to $\mu$ at $\mu=0$, where there is no sign problem 96. This is like taking the $\mu$-derivative of Fodor and Katz's reweighting factor, and including that in the observable. The relevant calculations have been performed by the Bielefeld/Swansea group [97. Their observables are the susceptibilities for the Polyakov loop and chiral condensate, the maxima of which indicate the position of the crossover line in the $\mu-T$ plane. Using improved gauge and staggered quark actions on a lattice of spacing $a \approx 0.2 \mathrm{fm}$, they obtain the results shown in Fig. 6. The results agree very well with the multiparameter reweighting lattice calculation, whose estimate of the critical endpoint is also shown. It remains to be seen whether this approach has advantages over multi-parameter reweighting. In 
particular, it is very hard to know how deep into the $\mu>0$ region the Taylor expansion around $\mu=0$ will continue to work. To the left of the dotted $\mu / T=0.4$ line in Fig. 6 the sign problem is under control, but the importance of higher order $\mu$-derivatives is yet to be checked.

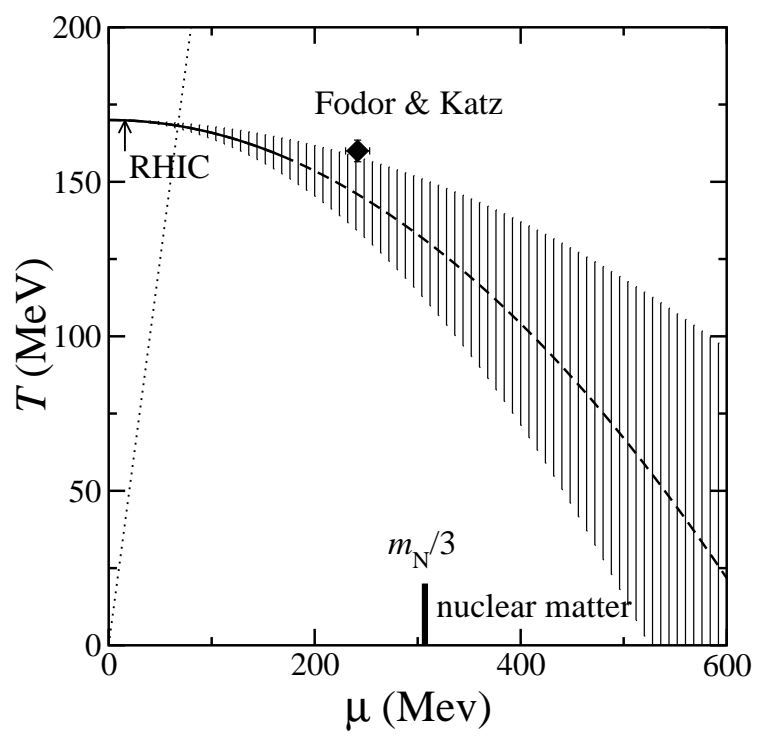

Figure 6. QCD hadronic/QGP crossover line from derivatives of susceptibilities with respect to $\mu$, evaluated at $\mu=0$ 97. Note the excellent agreement with Fodor and Katz's multiparameter reweighting calculation of the critical point $\left(\mu^{*}, T^{*}\right)$ (diamond).

\subsection{Imaginary $\mu$}

Over the years, various workers have pointed out that the sign problem only occurs for real chemical potential - the functional integral with imaginary $\mu$ is amenable to standard Monte-Carlo methods [98. Now full 4D calculations by de Forcrand and Philipsen 99. have yielded estimates of the position of the critical line in the $\mu-T$ plane for two-flavor QCD (for early explorations of 4 flavor QCD, see [100]). These authors perform calculations at several imaginary values of $\mu$, and then analytically continue to real $\mu$ by fitting their results to a power series in $\mu^{2}$, and extrapolating from negative to positive $\mu^{2}$ 101. The observables used to identify the crossover line were, as usual, the susceptibilities for the plaquette, Polyakov loop, and chiral condensate. Above the critical point $\left(\mu>\mu^{*}\right)$ there is a line in the $\mu-T$ plane along which these diverge in the large volume limit: this is the first-order line. Below the critical point $\left(\mu<\mu^{*}\right)$ each susceptibility shows a "ridge" of finite height in the large volume limit, and these ridges are expected to lie close together, approximately defining a narrow crossover strip. At large but finite volume, the line defined by the ridge/divergence of each susceptibility $\chi$ will be an analytic function $T_{\chi}(\mu)$. At infinite volume different susceptibilities will give the same line above $\mu^{*}$ but may give different ones below $\mu^{*}$ so it is not clear that the function remains analytic for all $\mu$, but at the volumes studied so far $T_{\chi}(\mu)$ turns out to be a very docile expansion in $\mu^{2}$ for imaginary $\mu$, fitting very well to the form $c_{0}+c_{1} \mu^{2}$ with no need for a $\mu^{4}$ term. The resultant crossover line for QCD with two light flavors is plotted in Fig. 7. It is consistent with the results from the other methods, and the errors are smaller.

Imaginary chemical potential is quite different from the two methods described above, and the errors appear to be well under control. It will be very interesting to see whether it can estimate the location of the critical point as accurately as it delineates the crossover region.

\subsection{Relevance to experiment}

It is very impressive that the different methods described above, all of which are being applied to QCD for the first time, achieve agreement in the position of the crossover line, and that there is even a rough estimate of the position of the critical point $\mu_{B}^{*} \sim 700 \mathrm{MeV}, T^{*} \sim 160 \mathrm{MeV}$. Putting aside caution and taking the numbers seriously for a moment, could such a critical point be accessible to heavy ion collision experiments? Treating the estimated value of $\mu_{B}^{*}$ as an upper limit, and bearing in mind that a heavy ion collision scans a range of temperatures down to the chemical freeze-out temperature $T_{\text {fo }}$ as the fire- 


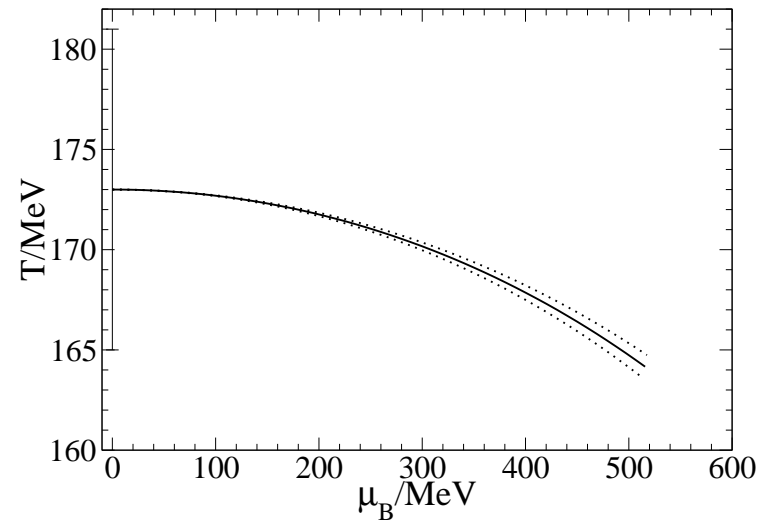

Figure 7. QCD hadronic/QGP crossover line from analytic continuation of imaginary $\mu$ results to real $\mu$ 99]. The result is consistent with Figs. 6, 5. Note that the horizontal axis is $\mu_{B}=3 \mu$.

ball expands, we can see from Table 1 that there is a chance that quark matter near the critical point could have been made at SPS. It may also be produced at the future experiment planned at GSI [102]. And now we can even envisage the possibility that theorists may predict the position of the critical point before experimentalists observe it.

\begin{tabular}{ccc}
\hline experiment & $\mu_{B}$ & $T_{\mathrm{fo}}$ \\
\hline RHIC & $40 \mathrm{MeV}$ & $170 \mathrm{MeV}$ \\
SPS & $250 \mathrm{MeV}$ & $170 \mathrm{MeV}$ \\
AGS & $550 \mathrm{MeV}$ & $130 \mathrm{MeV}$ \\
SIS & $700 \mathrm{MeV}$ & $80 \mathrm{MeV}$ \\
\hline
\end{tabular}

Table 1

Approximate chemical freeze-out temperatures and baryon chemical potentials for heavy-ion collisions at various colliders [103.

\section{Far from equilibrium}

One motivation for calculations of the QCD equation of state and transport properties at high temperature is their application to heavy ion collisions at current and future heavy-ion colliders. The success of hydrodynamic models of RHIC collisions 104 indicates that equilibrium is achieved within a very short time $\sim 1 \mathrm{fm} / c$, but perturbative estimates give longer timescales [105]. It is therefore very important to understand nonperturbative field theory far from equilibrium.

In the last two years there has been significant progress in treating non-equilibrium scalar field theory. It is based on systematic loop 107 and $1 / N$ 108,109 expansions of the twoparticle-irreducible (2PI) effective action formalism 63, 65 discussed in section 4.2. The formalism is illustrated diagramatically in Fig. 8. A typical mean-field calculation would only include the $\varnothing(N)$ tadpole (momentum-independent) contribution to the self-energy, and would not show thermal equilibration at long times. The NLO 2PI calculation also includes the $O(1)$ bubble chain contribution of Fig. 8, which is crucial for including rescattering effects that lead to equilibration.

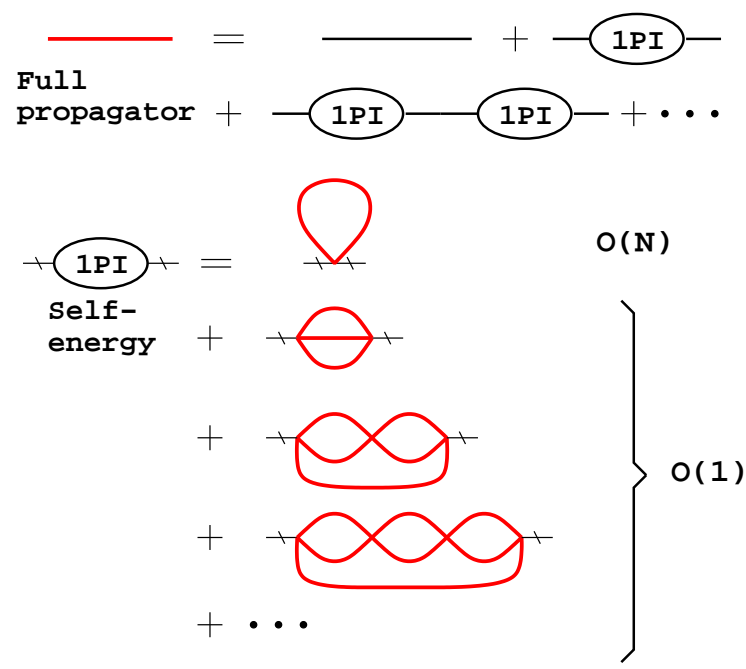

Figure 8. Coupled NLO Schwinger-Dyson equations for the full propagator from the 2PI effective action approach of [107]. 
Fig. 9 shows the time evolution of the propagator $G(p)$ in $1+1 \mathrm{D}$ scalar field theory, for three momentum modes $|p|=0,3,5$. The solid lines are for initial conditions close to the equilibrium value, the dashed lines for initial conditions further from the equilibrium value, and the dotdashed lines are for initial conditions far from the equilibrium value.

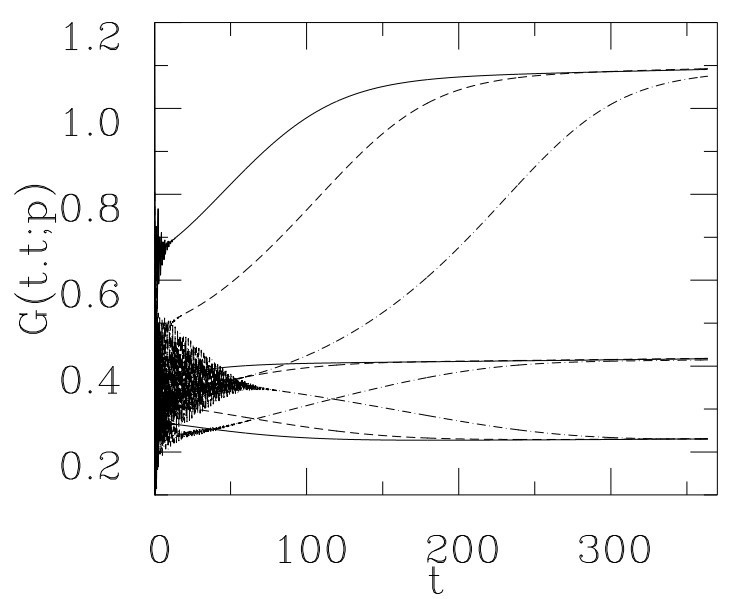

Figure 9. Time dependence of three modes of the equal time propagator in $1+1 \mathrm{D} \phi^{4}$ theory at non-zero temperature, for various initial conditions. Calculated by 3-loop 2PI effective action [108]. Note the quickly damped initial oscillations, followed by drifting and then comparably slow approach to equilibrium.

We see that the modes evolve in three stages,

1. Early: Initial oscillations quickly damped with rate $\gamma^{\text {(damp) }}$.

2. Intermediate: Drifting towards the equilibrium value with non-exponential/power-law behavior.

3. Late: Exponential approach to equilibrium, with rate $\gamma^{\text {(therm) }} \neq \gamma^{\text {(damp) }}$.
The rate of approach to equilibrium $\gamma^{\text {(therm) }}$ is found to be quite different from the initial damping rate $\gamma^{\text {(damp) }}$, and the overall equilibration time in scalar theories is clearly much longer than the microscopic timescales. Obviously it will be very interesting to push these calculations to higher dimensions [110] where spontaneous symmetry breaking can be studied, and to extend these methods to gauge theories, to see whether QCD predicts equilibration times as short as $1 \mathrm{fm} / c$ for the QGP.

\section{Conclusions}

There is great enthusiasm and activity among theorists studying high-temperature and highdensity QCD, stimulated by the successes of current heavy-ion experiments and the anticipation of future ones, and also by ongoing advances in the quality of observational data for neutron stars.

In high-density QCD, the upsurge of interest in color superconductivity is maturing into a effort to develop signatures for the presence of colorsuperconducting quark matter in compact stars. While ultra-high-density first-principles calculations and medium-density NJL calculations are becoming more sophisticated, useful results also follow from parameterizing the quark pairing with a gap parameter and studying its effects on phenomenologically important quantities.

In high-temperature QCD, lattice gauge theory is making continuous advances, achieving reliable calculations of equilibrium properties of the QGP such as screening lengths and the equation of state at temperatures up to a few times $T_{c}$. Dimensionally reduced lattice calculations will be able to extend this to arbitrarily high temperatures, when one remaining constant is computed. Various resummed perturbative calculations appear to agree with the lattice on the approximate size of deviations from the free equation of state above the critical temperature, although they are not yet on a solid independent footing. Complete leading-order perturbative expansions of transport properties, including all leading logs, are being achieved. The photon emission rate of the QGP has been computed, and other quantities 
will soon be calculated.

Lattice QCD has at last taken its first step into the non-zero-density region of the phase diagram. Remarkably, there is already agreement among the different methods on the position of the crossover line, and there is even a first estimate of the position of the chiral critical point, which is a sharply-defined physical observable. It would therefore be valuable for theorists, as well as striving for more accurate predictions of the position of the critical point, to further develop the existing work on signatures of the critical point in heavy-ion collisions.

Finally, field theories far from equilibrium are becoming accessible to controlled calculation. The 2PI effective action method has applications to disoriented chiral condensates, critical fluctuations, and other complexities of heavy ion collisions, as well as cosmological questions such as the exit from inflation in the early universe. Efforts to apply this method to higherdimensional scalar field theories, spontaneous symmetry breaking, and gauge theories are in progress.

Strong interaction physics at high temperature and density is a promised land that lies at the heart of modern physics, bordering on particle physics, nuclear physics, astrophysics, and condensed matter physics. Our first explorations are revealing the true extent of its fertility and richness.

\section{REFERENCES}

1. M. Gyulassy, nucl-th/0106072. U. W. Heinz, Nucl. Phys. A $\mathbf{6 8 5}$ (2001) 414 hepph/0009170.

2. M. G. Alford, Ann. Rev. Nucl. Part. Sci. 51 (2001) 131 hep-ph/0102047. K. Rajagopal and F. Wilczek, hep-ph/0011333. T. Schäfer and E. V. Shuryak, Lect. Notes Phys. 578 (2001) 203 nucl-th/0010049. D. K. Hong, Acta Phys. Polon. B 32 (2001) 1253 hepph/0101025. S. D. Hsu, hep-ph/0003140. D. H. Rischke and R. D. Pisarski, nuclth/0004016.

3. F. Karsch, Lect. Notes Phys. 583 (2002) 209 hep-lat/0106019. J. P. Blaizot and
E. Iancu, Phys. Rept. 359 (2002) 355 hep$\mathrm{ph} / 0101103$.

4. J. Berges and K. Rajagopal, Nucl. Phys. B538, 215 (1999) hep-ph/9804233.

5. M. Stephanov, K. Rajagopal and E. Shuryak, Phys. Rev. D60, 114028 (1999) hepph/9903292.

6. K. Rajagopal, E. Shuster, Phys. Rev. D62 085007 (2000) hep-ph/0004074.

7. P. Arnold and C. Zhai, Phys. Rev. D 50 (1994) 7603 hep-ph/9408276]; ibid. 51 (1995) 1906 hep-ph/9410360].

8. B. Barrois, Nucl. Phys. B129 (1977) 390; "Nonperturbative effects in dense quark matter", Cal Tech PhD thesis, UMI 79-04847mc (1979). S. Frautschi, Proceedings of workshop on hadronic matter at extreme density, Erice 1978. D. Bailin and A. Love, Phys. Rept. 107 (1984) 325, and references therein. M. Iwasaki, T. Iwado, Phys. Lett. B350, 163 (1995); M. Iwasaki, Prog. Theor. Phys. Suppl. 120, 187 (1995)

9. M. Alford, K. Rajagopal and F. Wilczek, Phys. Lett. B422, 247 (1998) hepph/9711395. R. Rapp, T. Schäfer, E. V. Shuryak and M. Velkovsky, Phys. Rev. Lett. 81, 53 (1998) hep-ph/9711396.

10. J. Bardeen, L. Cooper, J. Schrieffer, Phys. Rev. 106, 162 (1957); Phys. Rev. 108, 1175 (1957)

11. D. Son, Phys. Rev. D59, 094019 (1999). T. Schäfer, F. Wilczek, Phys. Rev. D60, 114033 (1999). R. Pisarski, D. Rischke, Phys. Rev. D61, 074017 (2000). D. Hong, Nucl. Phys. B582, 451 (2000) hepph/9905523]; Phys. Lett. B473, 118 (2000) hhep-ph/9812510]. R. Pisarski, D. Rischke, Phys. Rev. D61, 051501 (2000). D. Hong, V. Miransky, I. Shovkovy, L. Wijewardhana: Phys. Rev. D61, 056001 (2000); erratum Phys. Rev. D62, 059903 (2000). W. Brown, J. Liu, H. Ren, Phys. Rev. D61, 114012 (2000); Phys. Rev. D62, 054016 (2000); Phys. Rev. D62, 054013 (2000). S. Hsu, M. Schwetz, Nucl. Phys. B572, 211 (2000).

12. T. Schäfer, Nucl. Phys. B575, 269 (2000) hep-ph/9909574. I. Shovkovy, L. Wijewardhana, Phys. Lett. B470, 189 (1999). 
13. G. Carter, D. Diakonov, Phys. Rev. D60, 016004 (1999)

14. M. Alford, K. Rajagopal and F. Wilczek, Nucl. Phys. B537, 443 (1999) hep$\mathrm{ph} / 9804403$.

15. T. M. Schwarz, S. P. Klevansky and G. Papp, Phys. Rev. C 60 (1999) 055205 nucl-th/9903048. B. Vanderheyden and A. D. Jackson, Phys. Rev. D 62 (2000) 094010 hep-ph/0003150. F. Gastineau, R. Nebauer and J. Aichelin, Phys. Rev. C 65, 045204 (2002). B. O. Kerbikov, hep-ph/0106324. M. Huang, P. f. Zhuang and W. q. Chao, Phys. Rev. D 65 (2002) 076012 hepph/0112124. M. Oertel and M. Buballa, hepph/0202098, Nucl. Phys. A 703 (2002) 770 hep-ph/0109095.

16. D. N. Walters, hep-lat/0208011.

17. T. Schäfer, Phys. Rev. D62, 094007 (2000). M. Buballa, J. Hosek and M. Oertel, hepph/0204275. M. Alford, J. Bowers, J. Cheyne, G. Cowan, in preparation.

18. T. Schäfer, F. Wilczek, Phys. Rev. Lett. 82, 3956 (1999) hep-ph/9903503].

19. N. Evans, J. Hormuzdiar, S. Hsu, M. Schwetz: Nucl. Phys. B581, 391 (2000) hep$\mathrm{ph} / 9910313$.

20. M. Srednicki, L. Susskind, Nucl. Phys. B187, $93(1981)$

21. M. Alford, J. Berges and K. Rajagopal, Nucl. Phys. B558, 219 (1999) hep-ph/9903502.

22. M. Alford and K. Rajagopal, JHEP 0206 (2002) 031 hep-ph/0204001.

23. P. F. Bedaque and T. Schäfer, Nucl. Phys. A 697 (2002) 802 hep-ph/0105150].

24. K. Iida and G. Baym, Phys. Rev. D 63, 074018 (2001) hep-ph/0011229.

25. K. Rajagopal and F. Wilczek, Phys. Rev. Lett. 86, 3492 (2001) hep-ph/0012039.

26. A. W. Steiner, S. Reddy and M. Prakash, hepph/0205201.

27. S. L. Shapiro and S. A. Teukolsky, "Black Holes, White Dwarfs, And Neutron Stars: The Physics Of Compact Objects," New York, USA: Wiley (1983) 645 p. N. K. Glendenning, "Compact Stars: Nuclear Physics, Particle Physics, And General Relativity," New York, USA: Springer (1997) 390 p.
J. M. Lattimer and M. Prakash, Phys. Rept. 333 (2000) 121 astro-ph/0002203. H. Heiselberg and V. Pandharipande, Ann. Rev. Nucl. Part. Sci. 50 (2000) 481 astro-ph/0003276.

28. M. Alford and S. Reddy, in preparation.

29. N. K. Glendenning, Phys. Rev. D 46, 1274 (1992).

30. S. Reddy, M. Sadzikowski and M. Tachibana, nucl-th/0203011.

31. S. Reddy, G. Bertsch and M. Prakash, coherent neutrino scattering," Phys. Lett. B 475, 1 (2000) nucl-th/9909040.

32. M. G. Alford, K. Rajagopal, S. Reddy and F. Wilczek, hep-ph/0105009.

33. A. I. Larkin and Yu. N. Ovchinnikov, Zh. Eksp. Teor. Fiz. 47, 1136 (1964) [Sov. Phys. JETP 20, 762 (1965)]; P. Fulde and R. A. Ferrell, Phys. Rev. 135, A550 (1964).

34. M. Alford, J. Bowers and K. Rajagopal, Phys. Rev. D 63, 074016 (2001) hep-ph/0008208.

35. R. Rapp, E. Shuryak, I. Zahed: Phys.Rev. D63 034008 (2001) hep-ph/0008207.

36. J. A. Bowers and K. Rajagopal, hep$\mathrm{ph} / 0204079$.

37. D. Pines, in "Neutron Stars: Theory and Observation", J. Venturaand, D. Pines, eds., 57 (Kluwer, 1991); M. Alpar. "Models for Pulsar Glitches", in The Lives of Neutron Stars, ed. by M. Alpar, U. Kiziloglu, J. van Paradijs (Kluwer, Dordrecht 1995) p 185; B. Link, R. Epstein, Astrophys. J. 457, 844 (1996); M. Ruderman, T. Zhu, K. Chen, Astrophys. J. 492, 267 (1998); A. Sedrakian, J. Cordes, Mon. Not. R. Astron. Soc. 307, 365 (1999)

38. D. Page, M. Prakash, J. Lattimer, A. Steiner, Phys. Rev. Lett. 85 (2000) 2048 hep$\mathrm{ph} / 0005094$.

39. D. G. Yakovlev, A. D. Kaminker, O. Y. Gnedin and P. Haensel, Phys. Rept. 354 (2001) 1 [astro-ph/0012122].

40. S. Tsuruta et al, astro-ph/0204508.

41. D. Blaschke, H. Grigorian, D.N. Voskresensky, Astron. Astrophys. 368 (2001) 561 [astro-ph/0009120]. D. Blaschke, T. Klahn, D. Voskresensky, Astrophys. J. 533, 406 (2000) astro-ph/9908334.

42. I. Shovkovy, P. Ellis aastro-ph/0207346].

43. D. Son, M. Stephanov, Phys. Rev. D61, 
074012 (2000); M. Rho, A. Wirzba, I. Zahed, Phys. Lett. B473, 126 (2000); R. Casalbuoni, R. Gatto, hep-ph/9911223, unpublished; D. Hong, T. Lee, D. Min, Phys. Lett. B477, 137 (2000); C. Manuel, M. Tytgat, Phys. Lett. B479, 190 (2000); S. Beane, P. Bedaque, M. Savage, Phys. Lett. B483, 131 (2000)

44. P. Jaikumar, M. Prakash and T. Schafer, astro-ph/0203088.

45. G. W. Carter and S. Reddy, Phys. Rev. D 62, 103002 (2000) hep-ph/0005228.

46. N. Andersson, ApJ 502, 708 (1998), J. Friedman and S. Morsink, ApJ 502, 714 (1998).

47. J. L. Friedman and K. H. Lockitch, grqc/0102114.

48. L. Bildsten, G. Ushomirsky, astro $\mathrm{ph} / 9911155$.

49. J. Madsen Phys. Rev. Lett. 85, 10 (2000) astro-ph/9912418.

50. P. Amore, M. C. Birse, J. A. McGovern and N. R. Walet, hep-ph/0110267.

51. J. Madsen, Phys. Rev. Lett. 87 (2001) 172003 hep-ph/0108036.

52. M. M. Forbes and A. R. Zhitnitsky, Phys. Rev. D 65 (2002) 085009 hep-ph/0109173).

53. D. B. Kaplan and S. Reddy, hep-ph/0107265.

54. A. Kryjevski and T. Norsen, Phys. Rev. D 66 (2002) 034010 hep-ph/0202208].

55. T. Schäfer, Phys. Rev. D 65 (2002) 094033.

56. D. B. Kaplan and S. Reddy, Phys. Rev. Lett. 88 (2002) 132302 hep-ph/0109256. K. B. Buckley and A. R. Zhitnitsky, hep$\mathrm{ph} / 0204064$.

57. D. T. Son, M. A. Stephanov and A. R. Zhitnitsky, Phys. Rev. Lett. 86 (2001) 3955 hepph/0012041. D. T. Son, hep-ph/0108260.

58. F. Karsch and H. W. Wyld, Phys. Rev. D 35 (1987) 2518. M. Asakawa, T. Hatsuda and Y. Nakahara, Prog. Part. Nucl. Phys. 46 (2001) 459 [hep-lat/0011040]. T. Yamazaki et al. [CP-PACS Collaboration], Phys. Rev. D 65 (2002) 014501 hep-lat/0105030.

59. F. Karsch, E. Laermann and A. Peikert, Phys. Lett. B478, 447 (2000)

60. A. Ali Khan et al. [CP-PACS Collaboration], action," Phys. Rev. D 63 (2001) 034502 heplat/0008011.
61. C. Zhai and B. Kastening, Phys. Rev. D 52 (1995) 7232 hep-ph/9507380].

62. E. Braaten and A. Nieto, Phys. Rev. D 53 (1996) 3421 hep-ph/9510408].

63. J. M. Cornwall, R. Jackiw and E. Tomboulis, Phys. Rev. D 10 (1974) 2428.

64. B. A. Freedman and L. D. McLerran, Phys. Rev. D 16, 1169 (1977).

65. J.M. Luttinger and J.C. Ward, Phys. Rev. 118 (1960) 1417; G. Baym, Phys. Rev. 127 (1962) 1391.

66. J. P. Blaizot, E. Iancu and A. Rebhan, Phys. Rev. D 63 (2001) 065003 hepph/0005003; Nucl. Phys. A 698 (2002) 404 hep-ph/0104033.

67. A. Peshier, Phys. Rev. D 63, 105004 (2001).

68. J. O. Andersen, E. Braaten, E. Petitgirard and M. Strickland, hep-ph/0205085.

69. G. Boyd et al., Phys. Rev. Let. 75, 4169 (1995), Nucl. Phys. B469, 419 (1996). M. Okamoto et al. (CP-PACS), Phys. Rev. D60, 094510 (1999).

70. A. Rebhan, personal communication. To appear in R. C. Hwa, "Quark - Gluon Plasma. Vol. 3," Singapore, Singapore: World Scientific (in preparation).

71. J. Kapusta, P. Lichard and D. Seibert, Phys. Rev. D 44, 2774 (1991) [Erratum-ibid. D 47, 4171 (1991)].

72. R. Baier, H. Nakkagawa, A. Niegawa and K. Redlich, Z. Phys. C 53, 433 (1992).

73. P. Aurenche, F. Gelis, R. Kobes and H. Zaraket, Phys. Rev. D 58, 085003 (1998) hep-ph/9804224.

74. P. Arnold, G. D. Moore and L. G. Yaffe, JHEP 0112 (2001) 009 hep-ph/0111107.

75. P. Aurenche, F. Gelis, R. Kobes and E. Petitgirard, Phys. Rev. D 54, 5274 (1996) hep$\mathrm{ph} / 9604398$.

76. P. Ginsparg, Nucl. Phys. B 170 (1980) 388; T. Appelquist and R.D. Pisarski, Phys. Rev. D 23 (1981) 2305.

77. S. Nadkarni, Phys. Rev. Lett. 60 (1988) 491; T. Reisz, Z. Phys. C 53 (1992) 169; L. Kärkkäinen et al, Phys. Lett. B 282 (1992) 121; Nucl. Phys. B 418 (1994) 3 heplat/9310014; Nucl. Phys. B 395 (1993) 733.

78. S. Huang and M. Lissia, Nucl. Phys. B 438 
(1995) 54 hep-ph/9411293; ibid. 480 (1996) 623 hep-ph/9511383].

79. K. Kajantie et al, Nucl. Phys. B 458 (1996) 90 hep-ph/9508379;; Phys. Lett. B 423 (1998) 137 [hep-ph/9710538].

80. K. Kajantie, M. Laine, K. Rummukainen and M. Shaposhnikov, Nucl. Phys. B 503 (1997) 357 hep-ph/9704416.

81. A.D. Linde, Phys. Lett. B 96 (1980) 289.

82. D.J. Gross, R.D. Pisarski and L.G. Yaffe, Rev. Mod. Phys. 53 (1981) 43.

83. K. Kajantie, M. Laine, K. Rummukainen and Y. Schroder, Phys. Rev. Lett. 86 (2001) 10 hep-ph/0007109.

84. A. Hart, M. Laine and O. Philipsen, Phys. Lett. B 505 (2001) 141 hep-lat/0010008.

85. J. Kogut, D. Sinclair, S. Hands and S. Morrison, Phys. Rev. D 64 (2001) 094505 heplat/0105026. J. Kogut, D. Toublan and D. Sinclair, hep-lat/0205019. I. Montvay, S. Hands, L. Scorzato and J. Skullerud, heplat/0112046.

86. J. B. Kogut and D. K. Sinclair, heplat/0202028.

87. S. Hands, B. Lucini and S. Morrison, Phys. Rev. D 65 (2002) 036004 hep-lat/0109001; Phys. Rev. Lett. 86 (2001) 753 heplat/0008027].

88. D. K. Hong and S. D. Hsu, hep-ph/0202236.

89. I. Barbour, C. Davies, Z. Sabeur, Phys. Lett. B215 (1988) 567. I. Barbour, S. Morrison, E. Klepfish, J. Kogut, M.-P. Lombardo, Nucl. Phys. (Proc. Suppl.) 60A (1998) 220.

90. M. G. Alford, S. Chandrasekharan, J. Cox and U. J. Wiese, Nucl. Phys. B 602 (2001) 61 hep-lat/0101012.

91. S. Chandrasekharan and U.-J. Wiese, Nucl. Phys. B492 (1997) 455. B. B. Beard, R. C. Brower, S. Chandrasekharan, D. Chen, A. Tsapalis and U.-J. Wiese, Nucl. Phys. Proc. Suppl. 63 (1998) 775. R. Brower, S. Chandrasekharan and U.-J. Wiese, Phys. Rev. D 60 (1999) 094502. O. Bar, R. Brower, B. Schlittgen and U. J. Wiese, Nucl. Phys. Proc. Suppl. 106 (2002) 1019 heplat/0110148.

92. Z. Fodor and S. D. Katz, JHEP 0203 (2002) 014 hep-lat/0106002];
93. Z. Fodor and S. D. Katz, Phys. Lett. B 534 (2002) 87 hep-lat/0104001.

94. Z. Fodor, S. D. Katz and K. K. Szabo, heplat/0208078.

95. M. A. Halasz, A. D. Jackson, R. E. Shrock, M. A. Stephanov and J. J. Verbaarschot, Phys. Rev. D 58 (1998) 096007 hep$\mathrm{ph} / 9804290$ ].

96. P. de Forcrand et al. [QCD-TARO Collaboration], Nucl. Phys. Proc. Suppl. 83 (2000) 408 hep-lat/9911034. S. Gottlieb, W. Liu, D. Toussaint, R. L. Renken and R. L. Sugar, Phys. Rev. D 38 (1988) 2888.

97. C. R. Allton et al., hep-lat/0204010.

98. N. Weiss, Phys. Rev. D35, (1987) 2495. E. Dagotto, A. Moreo, R. Sugar, D. Toussaint, Phys. Rev. B41, 811 (1990). A. Hasenfratz and D. Toussaint, Nucl. Phys. B371, 539 (1992). M. G. Alford, A. Kapustin and F. Wilczek, Phys. Rev. D 59 (1999) 054502 [hep-lat/9807039]. A. Hart, M. Laine and O. Philipsen, Nucl. Phys. B 586 (2000) 443 hep-ph/0004060.

99. P. de Forcrand and O. Philipsen, heplat/0205016.

100.M. D'Elia and M. P. Lombardo, heplat/0205022.

101.M. P. Lombardo, Nucl. Phys. Proc. Suppl. 83 (2000) 375 hep-lat/9908006.

102.Conceptual Design Report "An International Accelerator Facility for Beams of Ions and Antiprotons", GSI Darmstadt, 2001. http://www.gsi.de/GSI-Future/cdr/

103.P. Braun-Munzinger, Nucl. Phys. A 681 (2001) 119 nucl-ex/0007021.

104.U. W. Heinz and P. F. Kolb, hep-ph/0204061.

105R. Baier, A. H. Mueller, D. Schiff and D. T. Son, Phys. Lett. B 502 (2001) 51 hepph/0009237]. A. H. Mueller, Nucl. Phys. A $702(2002) 65$.

106.J. Manjavidze and A. Sissakian, Phys. Rept. 346 (2001) 1 hep-ph/0105245.

107.J. Berges and J. Cox, Phys. Lett. B 517 (2001) 369 hep-ph/0006160.

108.J. Berges, Nucl. Phys. A 699, 847 (2002)

109.G. Aarts, D. Ahrensmeier, R. Baier, J. Berges and J. Serreau, hep-ph/0201308.

110.J. Berges and J. Serreau, hep-ph/0208070. 